\title{
Semi-flexible interacting self-avoiding trails on the square lattice
}

\author{
A Bedini ${ }^{1}$, A L Owczarek ${ }^{1}$ and T Prellberg ${ }^{2}$ \\ ${ }^{1}$ Department of Mathematics and Statistics, \\ The University of Melbourne, Parkville, Vic 3010, Australia. \\ \{abedini, owczarek\}@unimelb.edu.au \\ 2 School of Mathematical Sciences \\ Queen Mary University of London \\ Mile End Road, London E1 4NS, UK \\ t.prellberg@qmul.ac.uk
}

March 7, 2022

\begin{abstract}
Self-avoiding walks self-interacting via nearest neighbours (ISAW) and self-avoiding trails interacting via multiply-visited sites (ISAT) are two models of the polymer collapse transition of a polymer in dilute solution. On the square lattice it has been established numerically that the collapse transition of each model lies in a different universality class.

It has been shown that by adding stiffness to the ISAW model a second low temperature phase eventuates and a more complicated phase diagram ensues with three types of transition that meet at a multi-critical point. For large enough stiffness the collapse transition becomes first-order. Interestingly, a phase diagram of a similar structure has been seen to occur in an extended ISAT model on the triangular lattice without stiffness. It is therefore of interest to see the effect of adding stiffness to the ISAT model.

We have studied by computer simulation a generalised model of self-interacting self-avoiding trails on the square lattice with a stiffness parameter added. Intriguingly, we find that stiffness does not change the order of the collapse transition for ISAT on the square lattice for a very wide range of stiffness weights. While at the lengths considered there are clear bimodal distributions for very large stiffness, our numerical evidence strongly suggests that these are simply finite-size effects associated with a crossover to a first-order phase transition at infinite stiffness.
\end{abstract}




\section{Introduction}

The collapse transition of a polymer in a dilute solution has been a continuing focus of study in lattice statistical mechanics for decades [1, 2]. This transition describes the change in the scaling of the polymer with length that occurs as the temperature is lowered. At high temperatures the radius of gyration of polymer scales in a way swollen relative to a random walk: this is known as the excluded volume effect. At low temperatures a polymer condenses into dense, usually disordered, globule, with a much smaller radius of gyration. The interest in this phase transition has occurred both because of the motivation of physical systems but also because of the study of integrable cases [3, 4] of lattice models, that have proved especially fruitful in two dimensions. While the canonical lattice model of the configurations of a polymer in solution has been the self-avoiding walk (SAW), where a random walk on a lattice is not allowed to visit a lattice site more than once, an alternative has been to use bond-avoiding walks, or a self-avoiding trail. A self-avoiding trail (SAT) is a lattice walk configuration where the excluded volume is obtained by preventing the walk from visiting the same bond, rather than the same site, more than once. These were used initially to model polymers with loops [5] but have subsequently occurred in integrable loop models in two dimensions [4]. A model of collapsing polymers can be constructed starting from self-avoiding trails, known as interacting self-avoiding trails (ISAT). Here energies are associated with multiply-visited sites and by favouring configurations with many such sites a collapse transition can be initiated.

Owczarek and Prellberg studied numerically the ISAT collapse on the square lattice by two different approaches [6, 7] and in either case found a strong continuous transition with specific heat exponent $\alpha=0.81(3)$. Recently, on the triangular lattice Doukas et al. [8] found that by changing the weighting of doubly and triply visited sites a first-order transition can ensure or alternatively, depending on the ratio of these weightings, a weaker second-order transition that mimics the collapse found in the canonical interacting self-avoiding walk (ISAW) model (also know as the $\theta$-point). They also found that the low temperature phase could become fully dense rather than globular.

Coorespondingly, there is also a modification of the ISAW model that displays two phase transitions for a range of parameters, namely the semi-flexible ISAW model [9, 10, 11]. Here two energies are included: the nearest-neighbour site interaction of the ISAW model and also a stiffness energy associated with consecutive parallel bonds of the walk (equivalently, a bending energy for bends in the walk). This has been studied on the cubic lattice by Bastolla and Grassberger [9]. They showed that when there is a strong energetic preference for straight segments, this model undergoes a single first-order transition from the excluded-volume high-temperature state to a fully dense state. On the other hand, if there is only a weak preference for straight segments, the polymer undergoes two phase transitions. On lowering the temperature the polymer undergoes a $\theta$-point transition to the liquid globule followed by a first-order transition to the fully dense phase at a lower temperature. Recent work by Krawczyk et al. [12] concerning the ISAW model on the square lattice in presence of a stiffness parameter showed that the introduction of stiffness can change the universality class of the collapse transition in two dimensions. For large stiffness the transition becomes first order and the collapsed 
phase moves from being globular to fully dense.

Recently, Foster [13] introduced and studied a generalised ISAT model on the square lattice which incorporates stiffness. Using transfer matrices and the phenomenological renormalisation group, that study predicted that the ISAT universality class is unaffected by a range of values of stiffness. However, the results suggested the appearance of a first-order transition for sufficiently large stiffness.

In this work we use Monte Carlo simulation to explore ISAT in presence of stiffness and the predictions of Foster [13]. We also explore the low temperature phase of the model and find that there is only one low temperature phase and that it is fully dense for the range of stiffness studied.

\section{ISAT}

The model of interacting trails on the square lattice is defined as follows. Consider the ensemble $\mathcal{T}_{n}$ of self-avoiding trails (SAT) of length $n$, that is, of all lattice paths of $n$ steps that can be formed on the square lattice such that they never visit the same bond more than once. Given a SAT $\psi_{n} \in \mathcal{T}_{n}$, we associate an energy $-\varepsilon_{t}$ with each doubly visited site, and denote their number by $m\left(\psi_{n}\right)$. The probability of $\psi_{n}$ is then given by

$$
\frac{e^{\beta \varepsilon_{t} m\left(\psi_{n}\right)}}{Z_{n}^{I S A T}(T)}
$$

where we define the Boltzmann weight $\omega_{t}=\exp \left(\beta \varepsilon_{t}\right)$ and $\beta$ is the inverse temperature $1 / k_{B} T$. The partition function of the ISAT model is given by

$$
Z_{n}^{I S A T}(T)=\sum_{\psi_{n} \in \mathcal{T}_{n}} \omega_{t}^{m\left(\psi_{n}\right)} .
$$

The finite-length reduced free energy is

$$
\kappa_{n}(T)=\frac{1}{n} \log Z_{n}(T)
$$

and the thermodynamic limit is obtained by taking the limit of large $n$, i.e.,

$$
\kappa(T)=\lim _{n \rightarrow \infty} \kappa_{n}(T)
$$

It is expected that there is a collapse phase transition at a temperature $T_{c}$ characterized by a nonanalyticity in $\kappa(T)$.

The collapse transition can be characterized via a change in the scaling of the size of the polymer with temperature. It is expected that some measure of the size, such as the radius of gyration or the mean squared distance of a monomer from the end points, $R_{n}^{2}(T)$, scales at fixed temperature as

$$
R_{n}^{2}(T) \sim A n^{2 \nu}
$$

with some exponent $\nu$. At high temperatures the polymer is swollen and in two dimensions it is accepted that $\nu=3 / 4[3]$. At low temperatures the polymer becomes dense in space, though not necessarily space filling, and the exponent is $\nu=1 / 2$. However, for the ISAT model the collapsed 
phase has been seen to be space filling [14. If the collapse transition is second-order, the scaling at $T_{c}$ of the size is intermediate between the high and low temperature forms. In the thermodynamic limit the expected singularity in the free energy can be seen in its second derivative (the specific heat). Denoting the (intensive) finite length specific heat per monomer by $c_{n}(T)$, the thermodynamic limit is given by the long length limit as

$$
C(T)=\lim _{n \rightarrow \infty} c_{n}(T) .
$$

One expects that the singular part of the specific heat behaves as

$$
C(T) \sim B\left|T_{c}-T\right|^{-\alpha},
$$

where $\alpha<1$ for a second-order phase transition. The singular part of the thermodynamic limit internal energy behaves as

$$
U(T) \sim B\left|T_{c}-T\right|^{1-\alpha},
$$

if the transition is second-order, and there is a jump in the internal energy if the transition is first-order (an effective value of $\alpha=1$ ).

Moreover, one expects crossover scaling forms [15] to apply around this temperature, so that

$$
c_{n}(T) \sim n^{\alpha \phi} \mathcal{C}\left(\left(T-T_{c}\right) n^{\phi}\right)
$$

with $0<\phi<1$ if the transition is second-order, and

$$
c_{n}(T) \sim n \mathcal{C}\left(\left(T-T_{c}\right) n\right)
$$

if the transition is first-order. From [15] we point out that the exponents $\alpha$ and $\phi$ are related via

$$
2-\alpha=\frac{1}{\phi} .
$$

Important for numerical estimation is the use of equation $(2.9)$ at the peak value of the specific heat given by $y^{\text {peak }}=\left(T-T_{c}\right) n^{\phi}$ so that

$$
c_{n}^{\text {peak }}(T) \sim \mathcal{C}^{\text {peak }} n^{\alpha \phi}
$$

where $\mathcal{C}^{\text {peak }}=\mathcal{C}\left(y^{\text {peak }}\right)$ is a constant.

A previous study [7] of ISAT model on the square lattice has shown that there is a collapse transition with a strongly divergent specific heat, with

$$
\alpha \phi=0.68(5)
$$

and so the individual exponents have been estimated as

$$
\phi=0.84(3) \quad \text { and } \quad \alpha=0.81(3) .
$$

At $T=T_{c}$, given by [16, 17]

$$
\omega_{t}=3,
$$

it was predicted [6] that

$$
R_{n}^{2}(T) \sim A n(\log n)^{2} .
$$




\section{Semi-flexible ISAT}

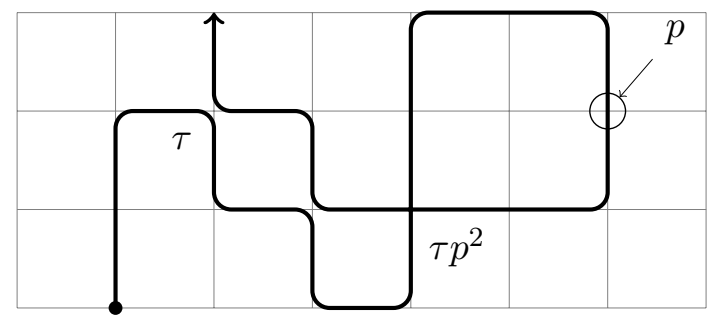

Figure 1: An example of semi-flexible ISAT configuration with three $(m=3)$ multiple-visit interactions associated with the Boltzmann weight $\tau$ and seven $(s=7)$ straight segments each associated with the Boltzmann weight $p$. Note that when the trail crosses itself, the crossing site is associated with a total weight $\tau p^{2}$ as a crossing necessarily requires two straight segments.

The semi-flexible ISAT (SFISAT) model can be defined as follows. Consider the set of bondavoiding paths $\mathcal{T}_{n}$ as defined in the previous section. Given a SAT $\psi_{n} \in \mathcal{T}_{n}$, we associate an energy $-\varepsilon_{t}$ every time the path visits the same site more than once, as in ISAT. Additionally, we define a straight segment of the trail by two consecutive parallel edges, and we associate an energy $-\varepsilon_{s}$ to each straight segment of trail.

For each configuration $\psi_{n} \in \mathcal{T}_{n}$ we count the number $m\left(\psi_{n}\right)$ of doubly-visited sites and $s\left(\psi_{n}\right)$ of straight segments: see Figure 1. Hence we associate with each configuration a Boltzmann weight $\tau^{m\left(\psi_{n}\right)} p^{s\left(\psi_{n}\right)}$ where $\tau=\exp \left(\beta \varepsilon_{t}\right), p=\exp \left(\beta \varepsilon_{s}\right)$, and $\beta$ is the inverse temperature $1 / k_{B} T$. The partition function of the SFISAT model is given by

$$
Z_{n}(\tau, p)=\sum_{\psi_{n} \in \mathcal{T}_{n}} \tau^{m\left(\psi_{n}\right)} p^{s\left(\psi_{n}\right)}
$$

The probability of a configuration $\psi_{n}$ is then

$$
p\left(\psi_{n} ; \tau, p\right)=\frac{\tau^{m\left(\psi_{n}\right)} p^{s\left(\psi_{n}\right)}}{Z_{n}(\tau, p)} .
$$

When we set $p=1$ the trail is fully flexible and the model reduces to the ISAT model. On the other end, if we set $p=0$ straight segments are excluded and our model requires the path to turn at every site: this is known as the "L-lattice". Trails on the L-lattice may be mapped [18 into the Interacting Self-Avoiding Walk model on the Manhattan lattice [19]. As a consequence of the mapping, the transition of ISAT on the L-lattice has been shown to be $\theta$-like with a convergent specific heat, unlike ISAT on the square lattice.

The average of any quantity $Q$ over the ensemble set of path $\mathcal{T}_{n}$ is given generically by

$$
\langle Q\rangle(n ; \tau, p)=\sum_{\psi_{n} \in \mathcal{T}_{n}} Q\left(\psi_{n}\right) p\left(\psi_{n} ; \tau, p\right) .
$$

In particular, we can define the average number of doubly-visited sites per site and their respective fluctuations as

$$
u=\frac{\langle m\rangle}{n} \quad \text { and } \quad c=\frac{\left\langle m^{2}\right\rangle-\langle m\rangle^{2}}{n} .
$$


One can also consider the average number of straight sections of the trail and their fluctuations

$$
u^{(s)}=\frac{\langle s\rangle}{n} \quad \text { and } \quad c^{(s)}=\frac{\left\langle s^{2}\right\rangle-\langle s\rangle^{2}}{n} .
$$

An important quantity for what follows is the proportion of the sites on the trail that are at lattice sites which are not doubly occupied:

$$
v_{n}=1-\frac{2\langle m\rangle}{n} .
$$

Foster [13] predicted that the universality class of fully flexible ISAT at $p=1$ extends to other values of $p$ and also that for large $p$ there may be a change to a first-order transition.

Defining the collapse as occurring at $\tau_{c}(p)$ for constant $p$, it follows that

$$
\tau_{c}(1)=3 \quad \text { and } \quad \tau_{c}(0)=2
$$

from the ISAT and L-lattice [18, 16, 17] results.

\section{Results}

We began by simulating the full two parameter space by using the flatPERM algorithm [20]. FlatPERM outputs an estimate $W_{n, \mathbf{k}}$ of the total weight of the walks of length $n$ at fixed values of some vector of quantities $\mathbf{k}=\left(k_{1}, k_{2}, \ldots, k_{\ell}\right)$. From the total weight one can access physical quantities over a broad range of temperatures through a simple weighted average, e.g.

$$
\langle\mathcal{O}\rangle_{n}(\tau)=\frac{\sum_{\mathbf{k}} \mathcal{O}_{n, \mathbf{k}}\left(\prod_{j} \tau_{j}^{k_{j}}\right) W_{n, \mathbf{k}}}{\sum_{\mathbf{k}}\left(\prod_{j} \tau_{j}^{k_{j}}\right) W_{n, \mathbf{k}}} .
$$

The quantities $k_{j}$ may be any subset of the physical parameters of the model. In our case we begin by using $k_{1}=m$ and $k_{2}=s$.

We have simulated SFISAT using the full two-parameter flatPERM algorithm up to length $n=512$, with $10^{5}$ iterations, collecting $7.8 \cdot 10^{9}$ samples at the maximum length.

To obtain a landscape of possible phase transitions we plot the largest eigenvalue of the matrix of second derivatives of the free energy with respect $\tau$ and $p$ (measuring the fluctuations and covariance in $m$ and $s$ ) at length $n=512$ in Figure 2 .

We notice that the strong peak corresponding to the ISAT transition at $(\tau, p)=(3,1)$ extends upward to larger values of $p$, becoming stronger as $p$ increases, as predicted in [13]. At smaller values of $p$ the peak of the specific heat seems to get weaker as it reaches the point $(\tau)=,(2,0)$. At this point, corresponding to a $\theta$-like transition, the specific heat is known to not diverge.

To investigate the nature of the transition at larger values of the stiffness parameter $p$, we have run extensive simulations of the model at few fixed values of $p$ all up to length $n=1024$. We have simulated different values of $p$ between $p=0.1$ and $p=10$, running between $S \simeq 2.3 \cdot 10^{6}$ and $S \simeq 4 \cdot 10^{6}$ iterations, and collecting between $6.0 \cdot 10^{9}$ and $1.7 \cdot 10^{10}$ samples at the maximum length. Following [20], we also measured the number of samples adjusted by the number of their independent growth 


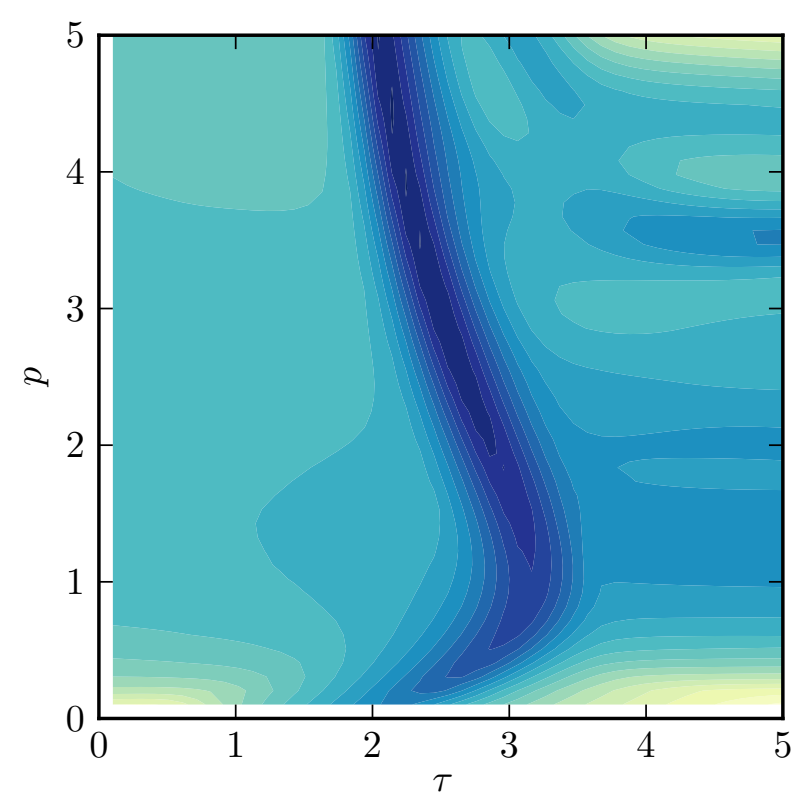

Figure 2: Density plot of the logarithm of the largest eigenvalue $\lambda_{\max }$ of the matrix of second derivatives of the free energy with respect to $\tau$ and $p$ at length 512. Darker regions denote larger values.

steps between $S^{e f f} \simeq 4.6 \cdot 10^{7}$ and $4.4 \cdot 10^{8}$ "effective samples" at the maximum length. Additionally, for the sake of comparison, we simulated the model at $p=0$ by putting the trails on the L-lattice, with $S=10^{6}$ iterations we collected $2.2 \cdot 10^{9}$ samples at the maximum length, corresponding to $7.4 \cdot 10^{7}$ effective samples.

\subsection{Specific heat}

We have begun by analysing the scaling of the specific heat by calculating the location of its peak $\tau_{n}^{*}=\arg \max _{\tau} c_{n}(\tau)$ and thereby evaluating $c_{n}^{*}=c_{n}\left(\tau_{n}^{*}\right)$. In Figure 3 , we plot the peak values of the specific heat for some of the models we have simulated. The exponent associated with the peak of the specific heat, see equation 2.12, is $\alpha \phi$ if the transition is second order. For $p=1$ we estimate $\alpha \phi \approx 0.64$ from trail lengths up to 1024 , which is a little less that our previous estimate of $0.68(5)$ based upon much longer length trails [7]. For $p>1$ we estimate $\alpha \phi \approx 0.74$ at $p=5$ and $\alpha \phi \approx 0.67$ at $p=10$, both of which are compatible with that previous estimate for the ISAT transition of 0.68(5). For $p=0$ we expect that the specific heat does not diverge, but at the lengths we consider it is not surprising to measure a weakly increasing specific heat peak with significant curvature in a log-log plot. We can then compare this to the situation when $p=0.5$. Although our estimate of $\alpha \phi \approx 0.52$ is somewhat smaller at $p=0.5$ than at $p=1$, it is still significantly larger than at $p=0$ and unless one is willing to postulate continuously changing exponents this suggests that the entire line up to (but excluding) $p=0$ is in the ISAT universality class. There could, of course, be curvature in the plot that we cannot observe at this length scale. However, we reinforce our conclusion by examining the low temperature phase below. 

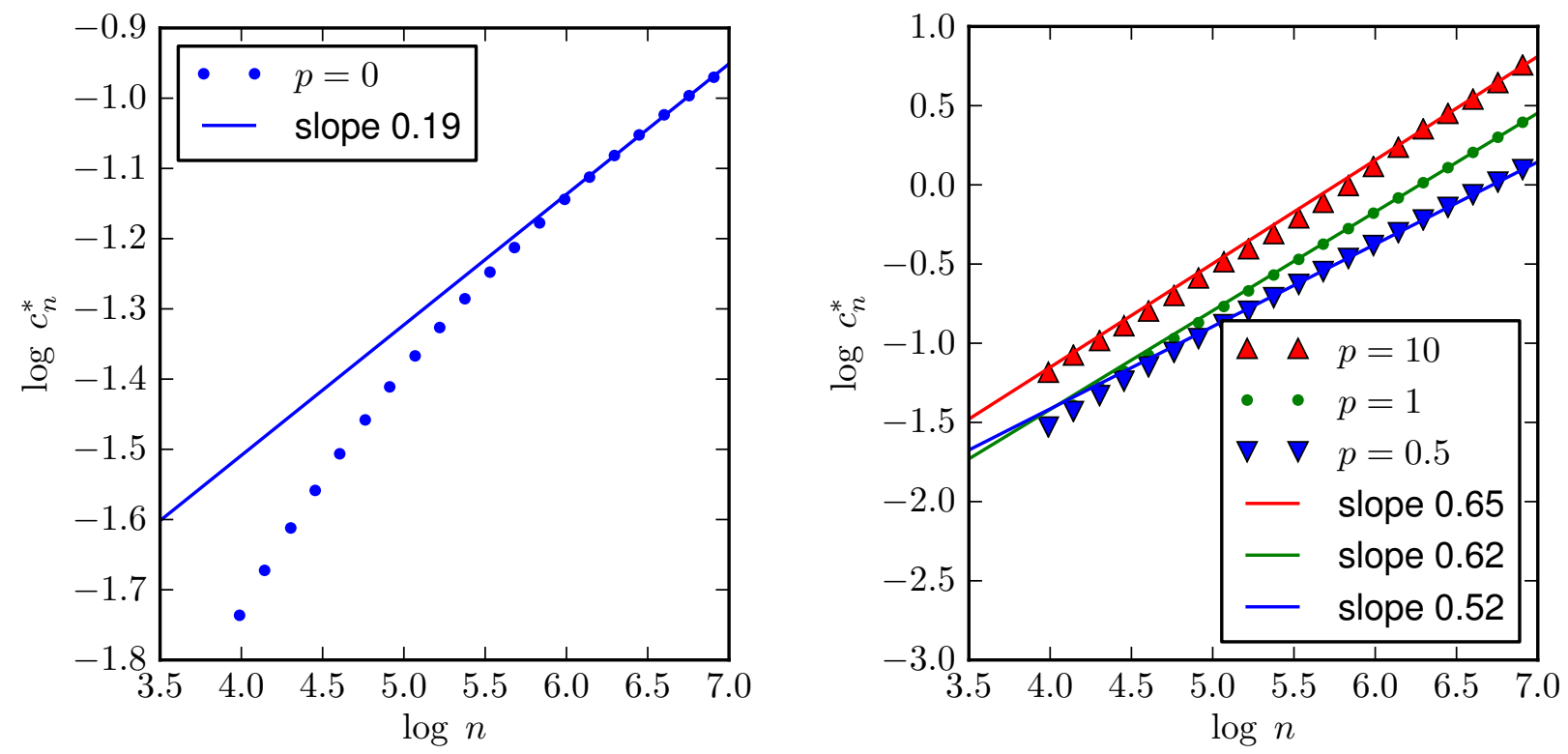

Figure 3: Plot of the logarithm of the peak value of the specific heat versus the logarithm of the length of the trails for $p=0$ (left) and $p=0.5,5,10$ (right). On the right, straight lines fit the data well, whereas there is clear curvature in the data on the left.

Before we consider the low temperature behaviour of our model, we also observe that the location of the transition in the thermodynamic limit, $\lim _{n \rightarrow \infty} \tau_{n}^{*}(p)=\tau_{c}(p)$, seems to obey

$$
1<\tau_{c}(p) \lesssim 3 \quad \text { for all } p
$$

attaining the value of $\tau_{c}(p)=3$ when $p=1$. As $p$ increases from zero, $\tau_{c}(p)$ increases until $p$ reaches some value near one, and then decreases again.

As indicated in Figure 4, for fixed large values of $p$, the location of the transition $\tau_{c}(p)$ is close to, but larger than one. We therefore conjecture that $\tau_{c}(p) \rightarrow 1$ as $p \rightarrow \infty$. The limit can be understood by the following ground state-type argument. For very large $p$ the ground state depends on whether $\tau$ is smaller or larger than one. For $\tau<1$ the ground state is a single straight rod, while for $\tau>1$ it is a configuration that fills every edge of the lattice in order to maximise doubly visited sites (that is, a set of crossing long rods that join up on the surface of the polymer). We point out that such an argument also naturally leads to a first-order transition at infinite $p$.

\subsection{Energy distribution}

We then investigated the claim [13, that for large finite values of $p$ the collapse transition becomes first-order. We did so by looking for the presence of bimodality in the energy distribution. In Figure 5 we plot the energy distribution in a neighbourhood of the critical point for $p=5$ and $p=10$. We don't find any evidence of a bimodality in either case.

On the other hand, we do find evidence of a double peak forming at very large values of $p$. In 


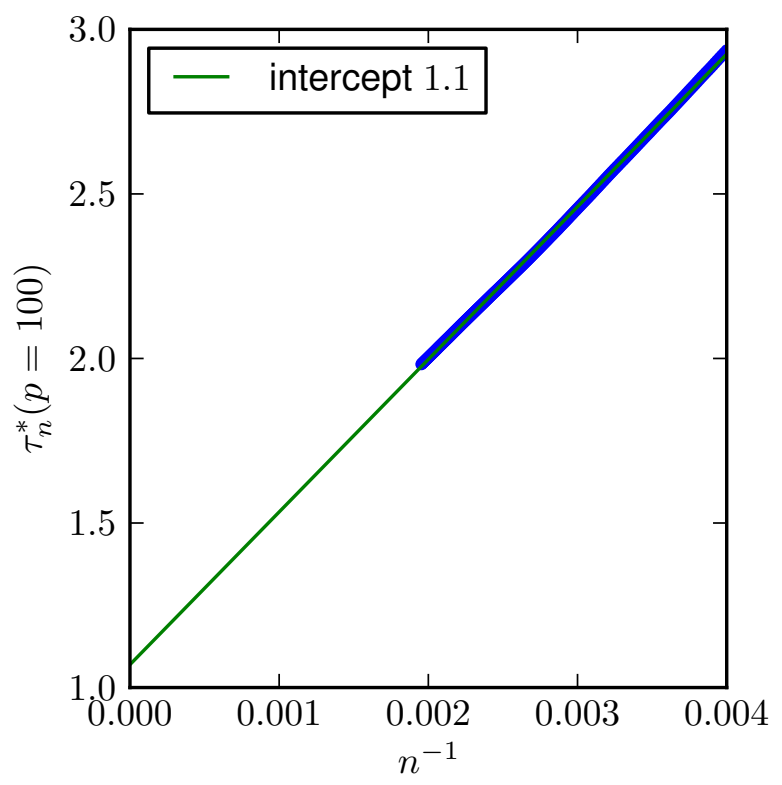

Figure 4: Plot of the location of the peak of the specific heat for $p=100$ versus inverse length $1 / n$. A simple linear extrapolation gives us the estimate $\tau_{c}(100) \approx 1.1$.

Figure 6 we plot the energy distribution near the transition for $p=100$, which has a clear double peak. Given the lengths of trails considered it is not clear immediately whether this is a manifestation of a crossover to an infinite $p$ behaviour, which we argued above may well be first-order, or a real change in the order of the collapse transition at finite $p$.

To investigate further we considered the smallest value of $p$ at which there is a non-zero latent heat at the transition point $\tau_{n}^{*}(p)$ as a function of $n$ : we denote this as $p_{1}(n)$. In Figure 7 we plot $p_{1}(n)$.

We immediately see that $p_{1}(n)$ increases in $n$. Hence, if the transition becomes first-order at some finite value of $p$ for infinite length trails, this would only occur for very large values of $p$. In fact, one can see that the increase in $p_{1}(n)$ is compatible with a power law such as $n^{1 / 2}$, which one would expect if the finite-size corrections were related to surface contributions. Additionally, we have considered the finite-size latent heat at fixed large $p$. An extrapolation against $n$ is compatible with a thermodynamic value of zero. This scenario would imply that we are simply seeing the crossover to the infinite- $p$ behaviour in the simulations, and that the transition in the thermodynamic limit of infinite length stays second-order for all finite values of $p$.

\subsection{Low temperature region}

There is strong evidence that the low-temperature phase of the ISAW model is a globular phase that is not fully dense, while for interacting trails the low-temperature phase is maximally dense [14], i.e., the trail fills the lattice asymptotically. Therefore, for trails the portion of steps not involved with doubly-visited sites should tend to zero as $n \rightarrow \infty$ in this phase. Following the analysis in [8, 14] we measured the proportion $v_{n}$ of steps not visiting the same site twice at $\tau=5$, corresponding 


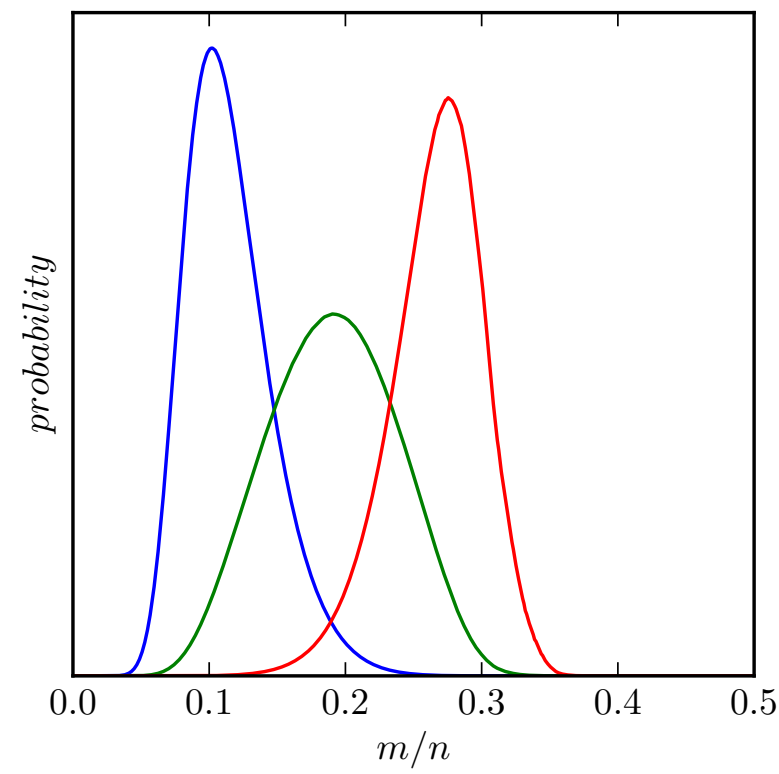

(a) $p=5$

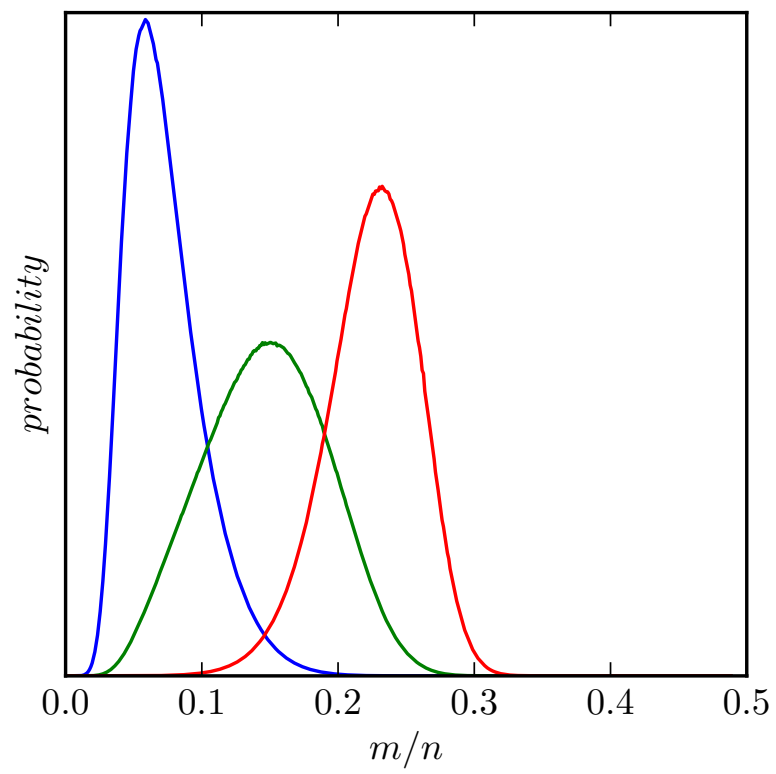

(b) $p=10$

Figure 5: Energy distribution across the critical region at length $n=1024$ for $p=5$ (a) and $p=10$ (b). For both figures the chosen temperatures are $\tau_{\text {left }}=1.85, \tau_{\text {middle }}=1.93$, and $\tau_{\text {right }}=2.02$. The weight $\tau_{\text {middle }}$ corresponds to the location of the specific heat peak at this length $\left(\tau_{n}^{*}\right.$ in the text), while $\tau_{\text {left }}$ and $\tau_{\text {right }}$ are given by $\tau_{\text {middle }} \pm \frac{1}{2} \Delta \tau_{n}$ where $\Delta \tau_{n}$ is the width of the specific heat peak at that length as measured at its half height. The distributions are normalised to have equal area.

to a sufficiently low temperature to be in the collapsed phase. Figure 8 shows a plot of $v_{n}$ against $n^{-1 / 2}$, clearly indicating that $v_{n} \rightarrow 0$ within error bars. This reinforces our earlier conclusion that for $0<p \leq 1$ the nature of collapse is that of the standard fully-flexible ISAT model, rather than changing to the ISAW-like $\theta$-transition that occurs when $p=0$.

\section{$5 \quad$ Phase diagram and Conclusions}

We have studied a generalised model of semi-flexible interacting trails (SFISAT) by including a stiffness parameter. From our analysis, we conjecture that the ISAT universality class is unaffected by the presence of stiffness. The universality class only changes in the singular limits of $p \rightarrow 0$ and $p \rightarrow \infty$. In the former limit the transition is $\theta$-like, whereas the transition turns first-order in the latter.

While at the lengths considered there are clear bimodal distributions for large values of $p$, our numerical evidence strongly suggests that these are likely to be finite-size effects associated with a crossover to a first-order phase transition at infinite stiffness.

Our results indicate that for all finite values of $p$ the low-temperature phase is maximally dense as in the fully-flexible ISAT model. Hence, unlike the ISAW model, there continues to be only a single low temperature phase when stiffness is added to ISAT. Putting the conclusions together suggests the 


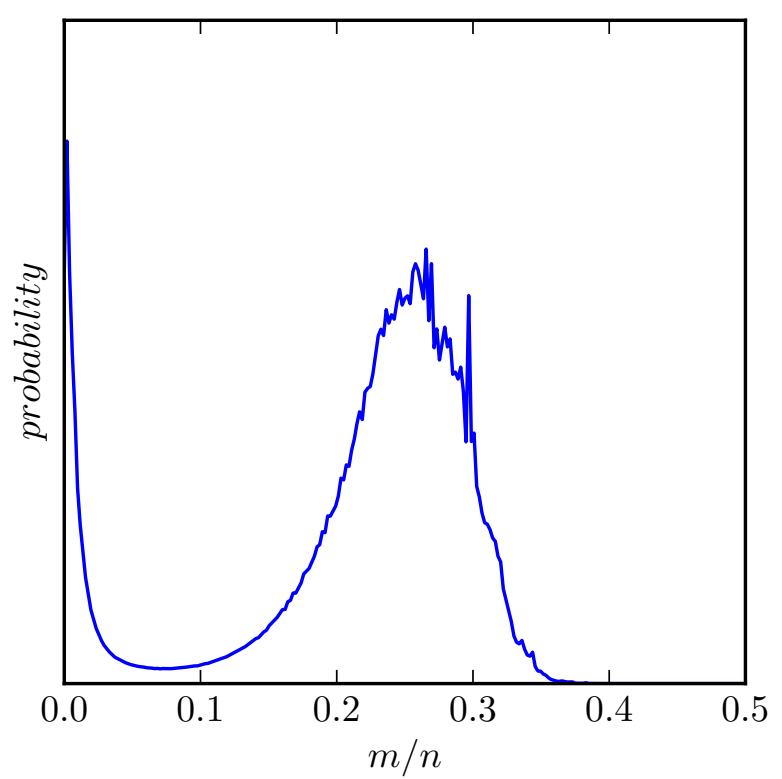

Figure 6: Energy distribution at length $n=512$ for $p=100$ at Boltzmann weight $\tau=2.01$, chosen so that the heights of the two peaks are roughly equal, very close to the specific heat peak located at $\tau^{*}=1.98$.

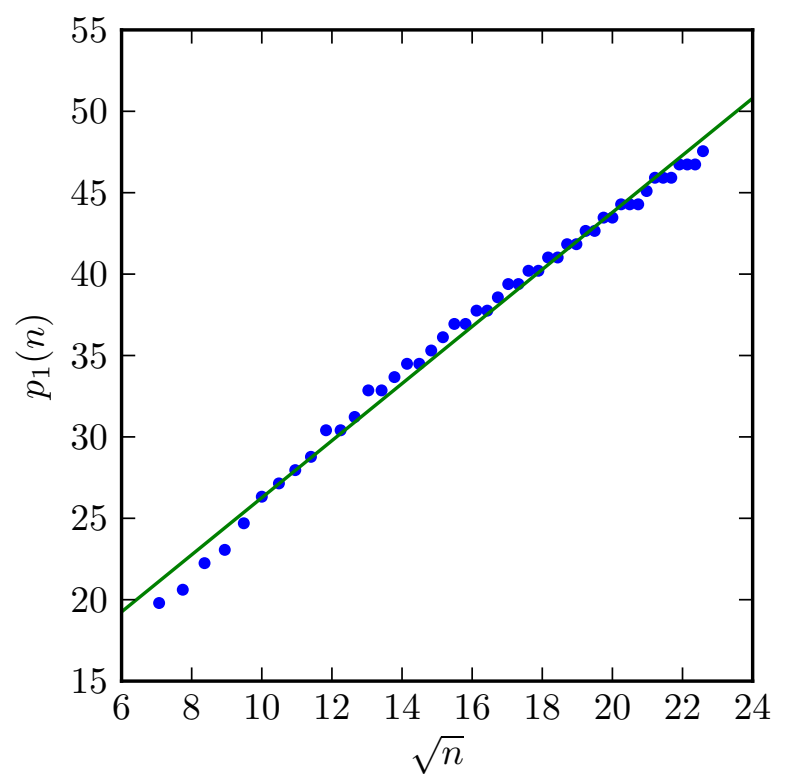

Figure 7: Plot of smallest $p$ value $p_{1}(n)$ for which an observable latent heat is encountered for a finite length trail, versus $\sqrt{n}$. This scale is chosen to reflect the possibility of finite-size corrections due to the presence of a surface in the bulk.

phase diagram shown in Figure 9.

It would be of some interest to examine the effect of stiffness on the canonical ISAT model on the 


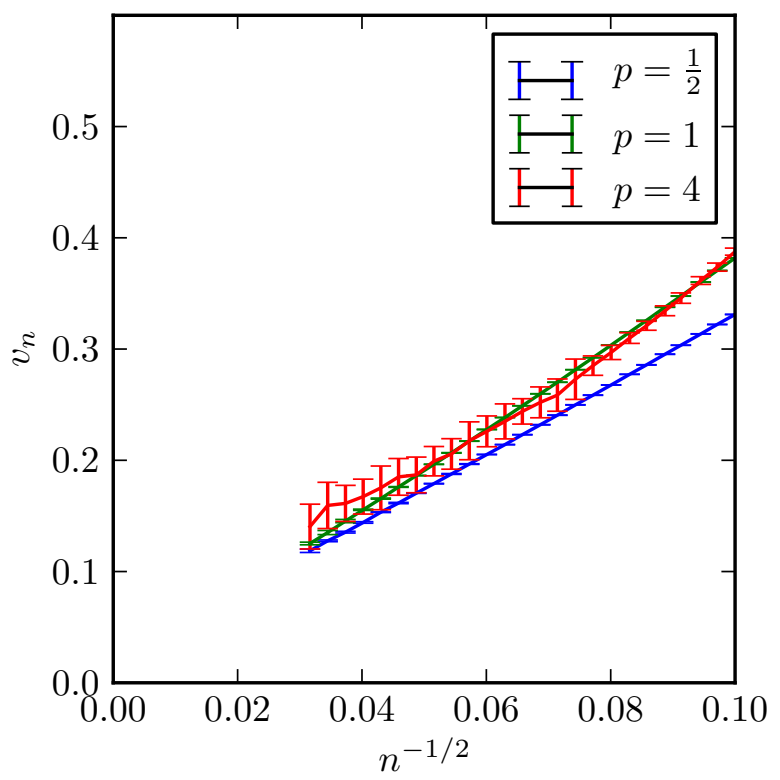

Figure 8: Plots of $v_{n}$, the proportion of steps visiting the same site once at $\tau=5$, which is in the low temperature phase, against $n^{-1 / 2}$. The scale $n^{-1 / 2}$ chosen is the natural low temperature scale.

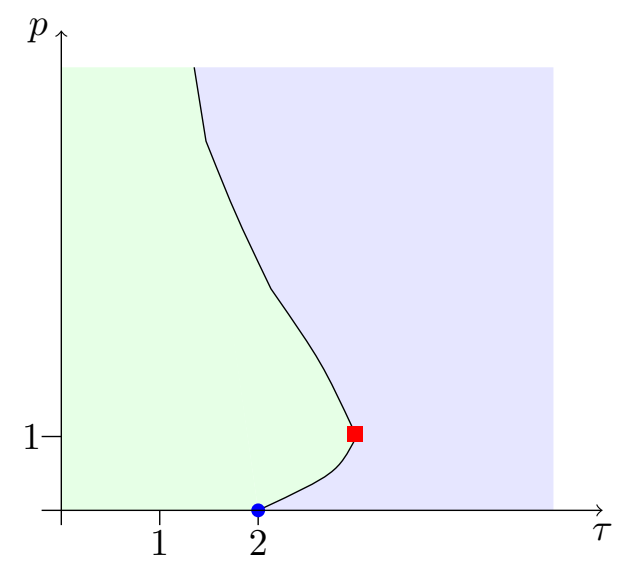

Figure 9: Schematic diagram of the SFISAT parameter space. The filled circle (blue online) on the horizontal axes depicts the location of the $\theta$-like transition at $(\tau, p)=(2,0)$, and the filled square (red online) depicts the location of the fully-flexible ISAT transition at $(\tau, p)=(3,1)$. The solid line corresponds to an ISAT-like transition. The whole region to the right of the curve, excluding the $p=0$ axis, is maximally-dense.

triangular lattice where the low temperature phase is globular rather than fully dense as it is on the square lattice considered in this paper. 


\section{Acknowledgements}

Financial support from the Australian Research Council via its support for the Centre of Excellence for Mathematics and Statistics of Complex Systems is gratefully acknowledged by the authors. The simulations were performed on the computational resources of the Victorian Partnership for Advanced Computing and the University of Melbourne High Performance Computing service. A L Owczarek thanks the School of Mathematical Sciences, Queen Mary, University of London for hospitality.

\section{References}

[1] P.-G. de Gennes, J. Physique Lett. 36, L55 (1975).

[2] P.-G. de Gennes, Scaling Concepts in Polymer Physics, Cornell University Press, Ithaca, 1979.

[3] B. Nienhuis, Phys. Rev. Lett. 49, 1062 (1982).

[4] S. O. Warnaar, M. T. Batchelor, and B. Nienhuis, J. Phys. A. 25, 3077 (1992).

[5] A. Malakis, Physica 84, 256 (1976).

[6] A. L. Owczarek and T. Prellberg, J. Stat. Phys. 79, 951 (1995).

[7] A. L. Owczarek and T. Prellberg, Physica A 373, 433 (2007).

[8] J. Doukas, A. L. Owczarek, and T. Prellberg, Phys. Rev. E 82, 031103 (12pp) (2010).

[9] U. Bastolla and P. Grassberger, J. Stat. Phys. 89, 1061 (1997).

[10] T. Vogel, M. Bachmann, and W. Janke, Phys. Rev. E 76, 061803 (2007).

[11] J. P. K. Doye, R. P. Sear, and D. Frenkel, J. Chem. Phys. 108, 2134 (1997).

[12] J. Krawczyk, A. Owczarek, and T. Prellberg, Physica A 389, 1619 (2010).

[13] D. P. Foster, Phys. Rev. E 84, 032102 (2011).

[14] A. Bedini, A. L. Owczarek, and T. Prellberg, arXiv:1210.7196 [cond-mat.stat-mech]

[15] R. Brak, A. L. Owczarek, and T. Prellberg, J. Phys. A. 26, 4565 (1993).

[16] H. Meirovitch, I. S. Chang, and Y. Shapir, Phys. Rev. A 40, 2879 (1989).

[17] R. M. Bradley, Phys. Rev. A 41, 914 (1990).

[18] R. M. Bradley, Phys. Rev. A 39, 3738 (1989).

[19] T. Prellberg and A. L. Owczarek, J. Phys. A. 27, 1811 (1994).

[20] T. Prellberg and J. Krawczyk, Phys. Rev. Lett. 92, 120602 (2004). 